\title{
》KADENZEN DES GANZEN KÖRPERS« - DAS SICH-BEWEGEN DES MUSIKERS
}

\author{
VERA VIEHÖVER UND STEPHAN WUNSCH
}

Über das Sich-Bewegen des Musikers zu sprechen scheint der Natur der Kunstform Musik nicht angemessen: Musik ist zum Hören da. Freilich: hörbare Musik entsteht nur, indem ein Musiker ein Instrument manipuliert (elektronisch erzeugte Klänge ausgenommen); indem der Körper des Musikers sich dem Klangkörper des Instruments zuwendet, es mit den Händen, den Füßen bedient, ihm mit den Lippen seinen Atem einbläst, es mit Schulter oder Knien stützt. Entsprechend verlangt der Klangkörper, dessen Form sich in einem oft Jahrhunderte währenden Prozess nach der Maßgabe optimaler Bedienbarkeit gebildet hat, seinerseits vom Musikerkörper, sich in eine dem Instrument angepasste Form zu bringen.

Die Optimierung der Bewegungsökonomie ist für Instrumentalisten praktisch aller Sparten Grundlage für die Verbesserung des Klangergebnisses. Ein Musikinstrument zu bedienen ist, mit Marcel Mauss zu sprechen, eine »Technik des Körpers«, mithin eine der »Weisen, in der sich die Menschen in der einen wie der anderen Gesellschaft traditionsgemäß ihres Körpers bedienen« (Mauss 1989: 199). ${ }^{1}$ Das Geformtwerden durch das Instrument, das als ausdifferenzierte Körpertechnik für den Betrachter erfahrbar wird, teilt der Musiker grundsätzlich mit jedem Menschen, der ein Werkzeug bedient. Zwar wird ein solcher Grad der Verfeinerung, wie er für die Ausführung virtuoser Musikstücke erforderlich ist, von einem Handwerker gewöhnlich nicht verlangt. Dennoch sieht man auch dem Benutzer einer Säge sein Können auf den ersten Blick an und wird es an der Qualität des Schnittes und der Schnelligkeit seiner Ausführung bestätigt finden. Der Sägenvirtuose wird das Werkzeug mit größter Gleichmäßigkeit führen, Druck und Zuggeschwindigkeit stehen im idea-

1 Mauss unterscheidet zwischen reinen »Techniken des Körpers« und »Techniken mit Instrumenten«, wobei er allerdings auch den Körper selbst schon als Instrument auffasst (206). Streng genommen müsste man in unserem Zusammenhang also von einer »Körpertechnik mit Instrument« sprechen. 
len Verhältnis, das Werkstück ist in der richtigen Höhe fixiert, Füße und die freie Hand verschaffen dem Handwerker einen sicheren Stand und erscheinen unbeweglich - der Körper des Sägenden ist auf das alleinige Ziel hin durchökonomisiert, dem ausführenden Arm eine ideale Kraftübermittlung zu gestatten. Nicht erwarten würde man hingegen, dass der Handwerker plötzlich mit der freien Hand Figuren in die Luft zeichnete, dass seine Lippen sich zu unhörbaren Lauten verformten, ein ekstatisches Pulsieren durch seinen Leib führe oder das bevorstehende Ende des Schnittes sich durch ein leidenschaftliches Vor- und Zurückwerfen des Kopfes ankündigte - und keinesfalls würde man solches Gebaren für ein Zeichen besonderer Professionalität halten.

Bei professionellen Musikern hingegen rufen solche Verhaltensweisen keineswegs Erstaunen hervor - im Gegenteil: Sie werden vom Publikum erwartet und mit gespannter Aufmerksamkeit verfolgt. Dabei sprechen wir nicht etwa von Rockmusikern, deren Live-Auftritte anderen Regeln gehorchen und deren Können in der Regel nicht primär in souveräner technischer Beherrschung eines Instrumentes besteht. Virtuosen der klassischen Musik leisten dagegen in technischer Hinsicht so Unglaubliches, dass man sich wundern muss, wie es ihrem Gehirn möglich ist, darüber hinaus noch Bewegungen zu gestalten, die außerhalb der Bewegungsökonomie stehen. Denn offenkundig dienen diese Bewegungen nicht der Manipulation des Instrumentes, ja müssten der effizienten Klanggestaltung eigentlich sogar abträglich sein. Ein Schaukeln des Oberkörpers - so möchte man meinen - kann die Aufgabe, auf einem lose auf der Schulter gelagerten Instrument Fingerbewegungen von höchster Schnelligkeit und Präzision auszuführen, nur erheblich erschweren. Ein energisches Zurückwerfen des Kopfes verringert die Nähe zu einem Tasteninstrument und müsste eigentlich der konzentrierten Spielbewegung schaden. Dennoch scheinen solche Bewegungen, die wir hier, weil sie spielökonomisch offenbar überflüssig sind, zunächst provisorisch »Restbewegungen« nennen wollen, dem Musizieren unmittelbar zuzugehören. Sie sind ganz offensichtlich nicht ohne weiteres vom Spielvorgang ablösbar und damit Teil des Sich-Bewegens des virtuosen Instrumentalmusikers. Wie können wir nun diese Bewegungen, deren Funktion in der bewegungsökonomischen Gleichung uns nicht unmittelbar einleuchtet, beschreiben? Handelt es sich hier um eine Art Gebärdensprache, die das rein musikalische Zeichensystem unterstützt, begleitet oder erweitert? Sind die »Restbewegungen« als Vehikel des Ausdrucks zu verstehen? Wenn ja, was drücken sie aus? 


\section{Domestizierung des Körpers}

Innerhalb der sogenannten >klassischen Musikkultur sind im Laufe der Jahrhunderte höchst unterschiedliche Körpertechniken entstanden, die jeweils einem eng umrissenen Kontext zugehören. So ist die Körpersprache des Orchestermusikers im Vergleich zu der des Solisten zurückgenommen; seine Bewegungen verlaufen zu denen des Pultnachbarn weitgehend synchron, und die im Gleichschritt auf- und niederfahrenden Bögen der Streicher im Orchester scheinen optisch das beste Zeugnis davon abzulegen, was einen >homogenen Klangkörper auszeichnet. Allzu auffällige Bewegungen würden beim Orchestermusiker schnell affektiert wirken und seine Einordnung ins Kollektiv in Frage stellen. Das SichBewegen der Musiker im professionellen Orchester hat einer am Ideal der Homogenität ausgerichteten Choreographie zu gehorchen. Die Mitglieder eines kammermusikalischen Ensembles bilden in der Regel wiederum andere Körpertechniken aus als die eines Symphonieorchesters. Häufig spielen sie stehend und nehmen mehr Bewegungsraum in Anspruch als ihre beengt sitzenden Kollegen aus dem Orchester. Der Ensemblespieler steht, was sein Bewegungsverhalten angeht, gewissermaBen zwischen dem Orchestermusiker und dem Instrumentalsolisten, auf den allein sich im Folgenden unsere Ausführungen beziehen.

Anders als Orchestermusiker und Ensemblespieler, deren Bewegungsradius allein schon durch den Körper des Nachbarn begrenzt wird, hat der Solist durch seine Bewegungen buchstäblich den eigenen »SpielRaum« zu definieren. Der Solist - so will es die grundsätzlich heute noch maßgebliche Tradition der bürgerlichen Konzertkultur des neunzehnten Jahrhunderts - tritt, insbesondere wenn er Virtuose ist, als einzigartiges Individuum auf, das durch eine die Grenzen des Gewöhnlichen sprengende künstlerische Begabung ausgezeichnet ist. Seine Darbietung erschöpft sich nicht im Hörbaren, sondern ist zugleich Schauspiel für das Auge. Seine häufig raumgreifenden, fast immer aber auffälligen Bewegungen beim Spiel gehören zum »Spektakel«. Sie beeinträchtigen den ästhetischen Genuss nicht - im Gegenteil: sie vermögen ihn gar zu verstärken. Diese wohlwollende Sicht auf den Instrumentalvirtuosen, der sein Sich-Bewegen geradezu ausstellt, ist allerdings recht neu. Dies wird deutlich, wenn man instrumentaldidaktische Texte aus dem 18. Jahrhundert daraufhin befragt, was sie den Schülern im Hinblick auf die »Techniken des Körpers« empfehlen.

Was wir »Rest« genannt haben, nämlich diejenigen Bewegungen des Musikers, die aus spielökonomischer Sicht überflüssig sind, da sie nicht zu den für die Klangerzeugung notwendigen Spielbewegungen gehören, haben offenbar bereits im 18. Jahrhundert das besondere Interesse der 
Musikpädagogen erweckt, bilden sie doch einen wichtigen Gegenstand der Instrumentalschulen der Aufklärung. Diese an Musiklehrer ebenso wie an Laienmusiker gerichteten, systematisch aufgebauten Lehrwerke traten im Zuge der allgemeinen Literalisierung allmählich an die Stelle der Etüdensammlungen oder Tabulaturen, die keine ausführlichen spieltechnischen Hinweise oder gar weitergehende Reflexionen über das $\mathrm{Mu}-$ sizieren als Kunst enthielten. Unter dem Titel L'ART DE TOUCHER LE CLAVECIN veröffentlichte im Jahr 1717 der Komponist und Cembalist François Couperin das »erste eigentliche Klavierlehrbuch« (Linde 1961: 7). Dort erfährt der Schüler:

"Sitzt man am Clavecin, so drehe man den Körper ein klein wenig nach rechts, die Knie seien nicht krampfhaft geschlossen, die Füße halte man nebeneinander, den rechten Fuß aber besonders nach außen. / Das Schneiden von Grimassen kann man sich selbst abgewöhnen, indem man einen Spiegel auf das Pult des Spinetts oder des Clavecin stellt. / [...] / Es ist besser und schicklicher, den Takt weder mit dem Kopfe, noch mit dem Körper oder den Füßen anzugeben. An seinem Clavecin soll man eine gefällige Miene zur Schau tragen. / Man hefte den Blick nicht starr auf einen bestimmten Gegenstand, schicke ihn aber auch nicht allzusehr ins Leere: endlich - man blicke die Gesellschaft, so eine vorhanden ist, an, als ob man gar nicht anderweitig beschäftigt wäre.« (Couperin 1961: 11)

Couperin hielt es demnach für unerlässlich, dem Schüler die Notwendigkeit der Selbstkontrolle nahezubringen. Nur solche Bewegungen sind erlaubt, die im Hinblick auf das Ziel der richtigen Tonerzeugung unabdingbar sind, alle anderen werden als »unschicklich « bewertet und sind deshalb rigoros auszumerzen. Die Forderung, eine »gefällige Miene« zu zeigen und den Blick nicht aufdringlich auf die »Gesellschaft« zu richten, verweist auf das Verhältnis zwischen Musiker und Publikum im frühaufklärerischen Musikbetrieb. Der Musiker, der normalerweise zur Unterhaltung adliger Gesellschaften spielt - und zwar nicht etwa auf einem Podium und auch nicht vor schweigenden Zuhörern -, gehört im weitesten Sinne zum Dienstpersonal. Er steht somit in der sozialen Hierarchie unter denen, die ihm zuhören, und ist nicht Mitglied ihrer Gesellschaft. Als Person soll der musikalische Lakai nicht in Erscheinung treten, und schon gar nicht soll er das Publikum mit den physischen Herstellungsbedingungen seiner Musik belästigen. Die »Restbewegungen« des Körpers, in denen der Musiker als Subjekt kenntlich wird, müssen daher so vollständig wie nur möglich zum Verschwinden gebracht werden.

Couperins didaktisches Werk ist ein Vorläufer der nur wenige Jahrzehnte später entstandenen, weitaus wirkungsmächtigeren Instrumentalschulen der Empfindsamkeit. Um die Mitte des 18. Jahrhunderts propa- 
gierten die empfindsamen Musikästhetiker die Abkehr vom musikalischen Mimesis-Prinzip. An die Stelle der bis dahin gültigen Vorstellung, die zentrale Aufgabe des Musikers bestehe darin, mit musikalischen Mitteln Affekte nachzuahmen, trat als neues Ideal der »Ausdruck« von Leidenschaften. Der Zuhörer sollte vom musikalischen Vortrag vor allem emotional gerührt werden, und Rührung, so liest man in Carl Philipp Emanuel Bachs Versuch ÜBer die WAHRE ART DAS Clavier ZU SPIELEN, konnte ein Musiker nur dann, wenn er selbst gerührt war. ${ }^{2}$ Aus diesem Empathie-Konzept ergibt sich für Bach auch eine neue Sicht auf die von Couperin noch pauschal für unerwünscht erklärten unwillkürlichen Körperbewegungen: Wenn der Musiker »dieselbe [!] Leidenschaften « empfinden soll, die »der Urheber eines fremden Stückes bey dessen Verfertigung hatte«, kann er gar nicht umhin, so meint Bach, seinen Körper ins Spiel zu bringen: $\gg \mathrm{Da} \beta$ alles dieses ohne die geringsten $\mathrm{Ge}-$ behrden abgehen könne, wird derjenige bloß läugnen, welcher durch seine Unempfindlichkeit genöthigt ist, wie ein geschnitztes Bild vor dem Instrumente zu sitzen. So unanständig und schädlich heßliche Gebährden sind: so nützlich sind die guten, indem sie unsern Absichten bey den $\mathrm{Zu}-$ hörern zu Hülfe kommen.« (122f.)

Bach hat seine im Vergleich zu Couperin mildere Sicht auf die »Gebährden« des Musikers in einer Zeit entwickelt, die dem Musiker einen neuen Status einräumte: Um die Mitte des 18. Jahrhunderts entwickelte sich in Konkurrenz zum adligen ein bürgerliches Musikleben, in dem der Musiker nicht mehr als Lakai auftrat, sondern als Freund unter Freunden. Das Verhältnis zwischen Musiker und Publikum wandelte sich also: Der Musiker wurde - zumindest in den kunstinteressierten bürgerlichen Kreisen - als vollwertiges Mitglied der Gemeinschaft akzeptiert. Aus Bachs Sicht ist es Aufgabe des Instrumentalisten, den Gleichklang der Gemüter herzustellen und als musikalischer Vermittler sogar den abwesenden Komponisten in den Kreis der Empfindsamen mit einzubeziehen.

Auch wenn in der Klavierschule im Hinblick auf den Umgang mit der Körperlichkeit des Musikers ein freierer Geist zu wehen scheint, darf doch nicht übersehen werden, dass auch Bach im Sinne der Bewegungseffizienz nutzlose Körperbewegungen nur nach Maßgabe einer strengen Ökonomie zulässt: Aus seiner Sicht sind sie Bestandteil des empfindsamen Ausdrucksverhaltens des Musikers und insofern notwendig, als sie letztlich nützlich sind: Sie dienen der optimalen Übertragung des musika-

2 »Indem ein Musickus nicht anders rühren kann, er sey dann selbst gerührt; so muß er nothwendig sich selbst in alle Affeckten setzen können, welche er bey seinen Zuhörern erregen will; er giebt ihnen seine Empfindungen zu verstehen und bewegt sie solchergestallt am besten zur Mit-Empfindung. « (Bach 1994: 122) 
lisch chiffrierten Sentiments vom Komponisten über die Schaltstelle Musiker auf den Zuhörer. Alle Entäußerungen des Körpers, die dieser - im Vergleich zur Zeit der Frühaufklärung immerhin erweiterten - Ökonomie des Musizierens nicht gehorchen, bleiben weiterhin tabu, denn sie verstoßen aus der Perspektive empfindsamer Ästhetik gegen die Regeln des »guten Geschmacks«. Verboten sind daher alle Arten von »Unarten«, wie es in Leopold Mozarts zur gleichen Zeit entstandenen GRÜNDLICHEN VIOLINSCHULE heißt, insbesondere

»das Bewegen der Violin; das hin und her Drehen des Leibes oder Kopfes; die Krümmung des Mundes oder das Rümpfen der Nase, sonderbar wenn etwas ein wenig schwer zu spielen ist; das Zischen, Pfeifen oder gar zu vernehmliche Schnauben mit dem Athem aus dem Munde, Halse oder Nase bey Abspielung einer oder der andern beschwerlichen Note; die gezwungenen und unnatürlichen Verdrehungen der rechten und linken Hand, sonderheitlich des Ellenbogens; und endlich die gewaltige Bewegung des Leibes, wodurch sich auch oft der Chor, oder das Zimmer wo man spielet erschüttert, und die Zuhörer bey dem Anblicke eines so mühsamen Holzhauers entweder zum Gelächter oder zum Mitleiden bewogen werden.« (Mozart 1995: 56f.)

$\mathrm{Zu}$ vermeiden ist jede Entgleisung, und zwar nicht nur, weil dadurch die Klangschönheit beeinträchtigt werden könnte, sondern weil unwillkürliche Körperbewegungen den ausführenden Musiker entblößen und der Peinlichkeit anheimgeben würden. Die peinlichen »Restbewegungen«, die Mozart aufzählt - Drehungen, Krümmungen, Zischen, Schnauben, Naserümpfen -, verweisen allesamt auf die tierische Natur des Menschen. Dieser freien Lauf zu lassen, würde eine Verletzung zentraler bürgerlicher Normen und damit letztlich den Ausschluss des Musikers aus der bürgerlichen Gesellschaft bedeuten. Der empfindsame Gefühlsausdruck muss deshalb paradoxerweise strengster rationaler Kontrolle unterliegen. ${ }^{3}$ Die zur Empfindsamkeit erzogene Seele des idealen Musikers bleibt damit im domestizierten Körper eingesperrt. »Reste « in der musikalischen Bewegungsgleichung sind weiterhin unerwünscht.

\section{Inszenierter Kontrollverlust}

Eine grundsätzlich veränderte Sicht auf das Sich-Bewegen des Musikers finden wir erst im 19. Jahrhundert, und zwar in den zeitgenössischen Zeugnissen über die Auftritte der großen Virtuosen, allen voran Franz Liszt und Niccolò Paganini. Die mediale Konstellation hat sich funda-

3 Zu diesem Paradoxon vgl. auch Kaden 1993: 143. 
mental geändert: Konzerte finden nicht mehr ausschließlich in adligen Salons und bürgerlichen Wohnräumen statt, sondern zunehmend in öffentlichen Sälen. Der Instrumentalsolist hat inzwischen das Podium erklommen. Dieser Wechsel in eine erhöhte Position ist insofern signifikant, als er wiederum ein verändertes Verhältnis zwischen Musiker und Publikum anzeigt: Der romantische Virtuose ist nicht mehr Teil der Gemeinschaft derer, die ihm zuhören; er spielt vielmehr in auratischer Ferne - auch dann, wenn er faktisch nicht mehr als einige Meter vom Publikum entfernt ist. Als Künstler steht der genialische Virtuose nun außerhalb der Gemeinschaft, die ihn als Idol bewundert und verehrt.

Im Übrigen ist auch das Medium, das uns heute über das SichBewegen der Virtuosen in Kenntnis setzt, ein anderes geworden: Waren bisher vornehmlich didaktische Werke unsere Quelle, so treten nun Augenzeugenberichte an deren Stelle. In ihnen berichten begeisterte Anhänger von genialischen Individuen, die das Konzert für das Publikum zu einem rauschhaften, singulären Ereignis zu machen vermögen - einem Ereignis nicht zuletzt für das Auge. Liest man die Berichte über die Auftritte des »Teufelsgeigers« Paganini, so fällt auf, dass über sein Aussehen und über seine Bewegungen mindestens ebenso viel reflektiert wird wie über das klangliche Ereignis seines Spiels. Immer wieder betonen die Zeitgenossen den Kontrast zwischen Paganinis kränklicher, ja fast leichenhafter Erscheinung und der Lebendigkeit seiner Bewegungen im Augenblick des Spiels. Dabei scheint sein Körper sich zu bewegen, ohne dass sein Geist noch in der Lage wäre, Kontrolle über ihn auszuüben. Max Julius Schottky schreibt:

$» \mathrm{Er}$ ist so mager, daß man nicht füglich magerer seyn kann [...]. Kaum scheint er in der Kleidung zusammenzuhängen, und macht er seine Verbeugungen, so bewegt sich der Körper auf eine sonderbare Art, daß man alle Augenblicke fürchtet, die Füße könnten sich vom Rumpfe trennen, und der ganze Mensch würde in einen Knochenhügel zusammenstürzen. Beim Spiel ist der rechte Fuß vorgeschoben, der, wenn die Passagen lebhafter werden, mit an's Komische grenzender Heftigkeit den Takt angiebt, ohne daß aber das Gesicht etwas von seiner Abgestorbenheit verlöre, außer wenn es sich für den Beifallsdonner zum sonderbaren Lächeln verzieht, wo sich allmählig die Lippen wunderlich verschieben und die Augen, zwar mit einigem Selbstgefühl, aber doch nicht ohne Gutmüthigkeit nach allen Seiten blinzeln. Bei schwierigen Stellen bildet sein Körper eine Art Dreieck, da sich der Leib dann übermäßig einbiegt während der Kopf und der rechte Fuß voranstehen.« (Schottky 1909: 2) [Hervorh. d. Verf.]

Paganinis Bewegungen scheinen sich im Spiel zu verselbständigen, die Glieder seines Körpers scheinen sich der Willkür des Musikers zu ent- 
ziehen: Nicht er bewegt sich, sondern seine Füße, sein Gesicht, seine Lippen, seine Augen bewegen ihn. Darüber hinaus gehorchen seine Bewegungen keiner spieltechnischen Ökonomie mehr: sie sind schlicht »übermäßig«. Dieses Übermäßige wird nun aber vom Publikum nicht mehr als anstößig empfunden, sondern im Gegenteil: Gerade in den Bewegungsexzessen, d.h. in der Überschreitung der Grenzen des Wohlanständigen und zugleich der spieltechnischen Ökonomie, erkennt es den untrüglichen Beweis dafür, dass hier das Ideal unbegrenzten Selbstausdrucks verwirklicht sei.

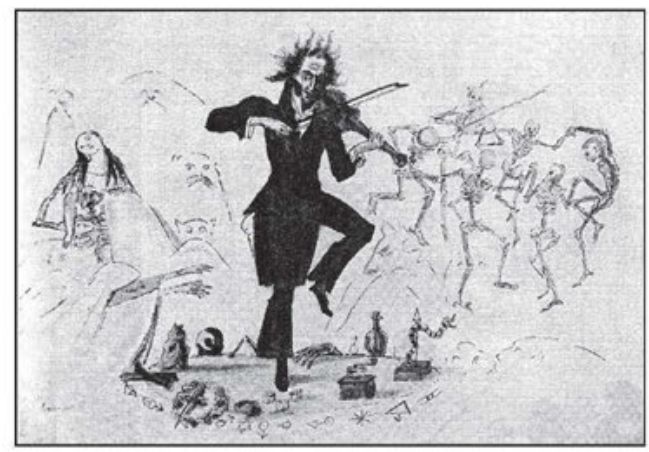

Abb. 1: DER TEUFELSGEIGER. Zeichnung von J. P. Lyser, Hamburg 1830

Für Johann Christian Lobe ist es denn auch die Fähigkeit, im Vortrag die eigene »Seele« zu geben, die Paganini einzigartig macht:

»Dieses Seelische ist es, [...] was seinem Tone jene eigentümliche Charakteristik gibt und deshalb unnachahmlich bleiben wird, weil er nur seine Seele reden läßt, nur sein Ich ausspricht. Er hat nämlich seine Violine zum Sprechorgan seiner innersten Empfindungen und seines eigentümlichen Gemüts- und Bildungszustandes gemacht. Was in seinem Innern vorgeht, drückt das Instrument mit seltener Wahrheit, Treue und Innigkeit aus [...].« (Lobe zit. nach Kapp 1969: 126f.)

Die Befreiung des Musikers aus dem Korsett der Bewegungsökonomie geht also einher mit der Entstehung einer neuen Vorstellung vom Künstler: Der Musiker als Virtuose ist demnach nicht mehr Diener der Musik, vielmehr dient die Musik ihm. Der wohlkalkulierte Kontrollverlust verhilft ihm, so will es die zugrunde liegende ästhetische Ideologie, zur vollkommenen Selbstaussprache. Der Zuhörer, der im romantischen Konzertbetrieb immer zugleich Zuschauer ist, wird dabei in die Position eines Voyeurs versetzt, der sich am Exhibitionismus des Künstlers berauscht. Gerade in den nicht mehr domestizierbaren Bewegungen, in der 
schamlosen Ausstellung von Körperlichkeit, offenbart sich ihm die Subjektivität des genialischen Individuums.

Dieses Phänomen lässt sich auch am Beispiel Franz Liszts nachvollziehen, der - abgesehen von Paganini - wie kein anderer den Typus des romantischen Virtuosen verkörpert. Für Dieter Hildebrandt, Autor eines populärwissenschaftlichen Standardwerks über die Geschichte des Klavierspiels im 19. Jahrhundert, ist Liszts außergewöhnliche Suggestionskraft in hohem Maße darauf zurückzuführen, dass es ihm gelungen sei, »das Klavier zum Schauplatz eines Dramas « zu machen, in dem »alle die Kämpfe, Leidenschaften, Naturgewalten und Todesritte« nicht allein in der Musik, sondern auch in Mimik und Gestik dargestellt würden: $» D a$ gibt es Gebärden bis hin zur Ungebärdigkeit, Sprünge nicht nur auf den Tasten, sondern auch Sprünge vom Stuhl, Windungen wie die einer Pythia, da wandert der Kopf immerzu hin und her wie der eines Zuschauers beim Tennis, da gibt es Kadenzen des ganzen Körpers [...], ArmEkstasen über donnernden Akkorden [...].« (Hildebrandt 2002: 207)

Wie die Auftritte Paganinis waren auch die Liszts für die Zeitgenossen in hohem Maße visuelle Ereignisse. So schreibt Heinrich Heine über den jungen Liszt: »Wenn er z. B. damals auf dem Pianoforte ein Gewitter spielte, sahen wir die Blitze über sein eigenes Gesicht dahinzucken, wie von Sturmwind schlotterten seine Glieder, und seine langen Haarzöpfe träuften gleichsam vom dargestellten Platzregen.« (Heine 1994: 425)

Für Robert Schumann, ein Augenzeuge wie Heine, ist das Bewegungsbild, das der Virtuose dem Zuschauer zeigt, gar integraler Teil der Ästhetik des Lisztschen Klavierspiels: »In Secundenfrist wechselt Zartes, Kühnes, Duftiges, Tolles: das Instrument glüht und sprüht unter seinem Meister. [...] Aber man muß das hören und auch sehen, Liszt dürfte durchaus nicht hinter den Coulissen spielen; ein großes Stück Poesie ginge dadurch verloren.« (Schumann 1914: 87) Schumann sieht bei Liszt das neue Ideal der Selbstaussprache des Musikers in reinster Form verwirklicht: »Es ist nicht mehr Clavierspiel dieser oder jener Art, sondern Aussprache eines kühnen Charakters überhaupt, dem zu herrschen, zu siegen das Geschick einmal statt gefährlichen Werkzeugs das friedliche der Kunst zugetheilt.« (Ebd.) 


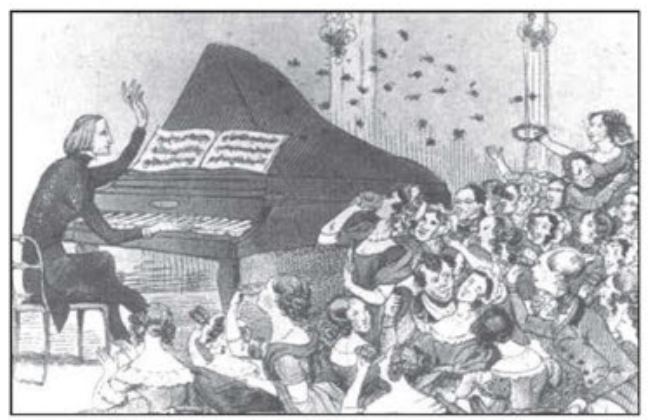

Abb. 2: Karikatur eines Liszt-Auftrittes (Berlin 1842)

Das exzessive Bewegungsspiel der großen Virtuosen Paganini und Liszt erleben die Zeitgenossen somit als Ausdruck im psychologischen Verständnis: Das, was wir »Rest« genannt haben, ist für den romantischen Zuhörer/Zuschauer nicht mehr, wie in der Epoche der Empfindsamkeit, unvermeidliche Begleiterscheinung bei der Übermittlung musikalischer Botschaften vom Komponisten über den Musiker zum Publikum, sondern Vehikel der »leidenschaftlich aufgestürmten Seele ${ }^{4}$ des Virtuosen. Die romantische Ideologie besagt: Gerade durch das Überflüssige, durch den aus der Bewegungsökonomie ausbrechenden Körper, »spricht« das Genie. Indem es den Kontrollverlust zum Ereignis macht, führt es den Zuschauern ihr Gefangensein in den Normen bürgerlicher Wohlanständigkeit vor. Sie werden Zeugen einer Entfesselung der Leidenschaften, die ihnen selbst nicht zusteht. Um so größer ist der voyeuristische Genuss.

\section{Glenn Gould auf DVD}

Wie eingangs schon erwähnt, gehorcht der heutige Konzertbetrieb und damit auch der Auftritt des Virtuosen theatralen Regeln, die im Wesentlichen in der ersten Hälfte des 19. Jahrhunderts ausgeformt worden sind (vgl. dazu auch Brandstetter 2002: 214). Das klassische Konzert von heute ist insofern nichts anderes als ein Zitat des 19. Jahrhunderts. Zugleich ist es für das Musikleben ein Nebenschauplatz geworden, während der Tonträgermarkt den eigentlichen Schauplatz darstellt. Die Allhörbarkeit der Musik im Zeitalter ihrer digitalen Brennbarkeit aber hat das Bedürfnis, den Musiker beim Spiel zu sehen, nicht gemindert, und der technische Fortschritt hat dafür gesorgt, dass uns der Musiker durch

4 Ludwig Rellstab: Franz Liszt. Berlin 1842. Zit. n. Dömling: 1985 
Film- und Fernsehaufnahmen, zunehmend auch über das Internet, heute so nah vor Augen gebracht wird wie nie zuvor. Dass in Zeiten eines immer härter werdenden Kampfes um die Aufmerksamkeit der Medien der Aspekt der kalkulierten Inszenierung des eigenen Bewegungsverhaltens eine immer größere Rolle spielt, versteht sich fast von selbst. So ist die Visualisierung stilisierter Bewegung inzwischen Bestandteil wohlausgefeilter Marketingkonzepte der jeweiligen Plattenfirmen.

Auffällig ist dabei, dass - wie hier im Falle des chinesischen KlavierPopstars Lang Lang - nicht selten gerade solche Bewegungsbilder ausgewählt werden, die den Musiker zwar in Bewegung, aber außerhalb des Spielvorgangs zeigen.

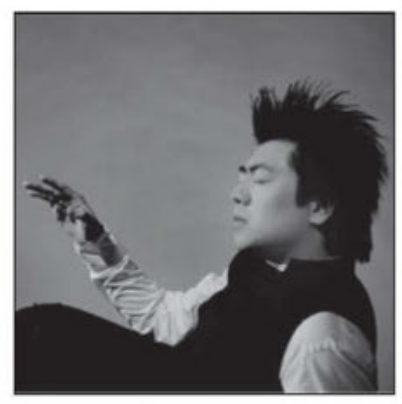

Abb. 3: Der chinesische Pianist Lang Lang

Auch wenn Rituale der Selbstinszenierung, auch der Inszenierung der eigenen Körperbewegungen, heute mehr denn je zuvor die Medienauftritte großer Virtuosen kennzeichnen, so hat sich doch insofern seit den Zeiten Liszts und Paganinis nicht viel geändert, als die Inszenierung im Kern immer noch auf dem Ausdrucksparadigma beruht: Wo ein ekstatisches Gebaren kalkuliert eingesetzt wird, geschieht es mit der Intention, das tradierte Stereotyp des genialischen Individuums beim Betrachter zu aktivieren. Insofern wird auf das Konzept »Bewegung als Ausdruck von Innerlichkeit« auch da Bezug genommen, wo Bewegung zum reinen Oberflächenphänomen geworden ist. Mag der Konsument auch heute nicht mehr in jedem überragenden Virtuosen ein Genie erkennen, so ist doch der Glaube geblieben, dass die mehr oder weniger ekstatischen Bewegungen, die die modernen Virtuosen kultivieren, eine besondere Sprache sind, in denen die Musiker sich selbst artikulieren.

Der legendäre kanadische Pianist Glenn Gould, dessen »Restbewegungen « wir im Folgenden genau zu beschreiben versuchen, hat die soeben skizzierte mediale Entwicklung früher als andere erkannt und mit großer Konsequenz in seiner eigenen Musizierpraxis vollzogen: 1964 
verkündete er seinen endgültigen Abschied vom öffentlichen Konzertpodium und war fortan nur noch via Schallplatteneinspielung für sein Publikum zu hören. Dieser Abschied lässt sich als bewusster Bruch mit der empfindsam-romantischen Tradition verstehen: Indem Gould freiwillig auf das Podium verzichtet, löst er auch sein Publikum auf und vollzieht die radikale Re-Privatisierung des Musizierens. Die Idee einer Kommunion der Seelen im gemeinsamen Musikerlebnis ist damit aufgekündigt. Gould zieht gewissermaßen eine Wand zwischen sich und dem Publikum auf.

Bekanntlich aber öffnete der angeblich so öffentlichkeitsscheue Gould sein Tonstudio bereitwillig für Kamerateams, die ihn bei seinen Einspielungen filmen sollten. So hatte die Öffentlichkeitsflucht des Eremiten Gould zur Folge, dass sein Spiel auch visuell bestens dokumentiert ist. Die Kamera inszeniert seine Spielbewegungen in Großaufnahme und zeigt sie in Einstellungen auf Hände, Gesicht, Profil und über die Schulter. Die mediale Konstellation, unter der wir uns dem Sich-Bewegen des Musikers anzunähern vermögen, hat sich damit ein weiteres Mal geändert: Während das Agieren Liszts und Paganinis uns wie den meisten Zeitgenossen allein durch schriftliche Berichte zugänglich ist und nur den unmittelbaren Augenzeugen als einmaliges, rauschhaftes Ereignis erfahrbar war, können wir Goulds Spiel jederzeit im Wohnzimmer betrachten, von allen Seiten, immer wieder, im schnellen Vor- und Rücklauf und - wie hier im Text - als Standbild. ${ }^{5}$

Auffällig im Hinblick auf Goulds »Technik des Körpers« ist schon die Art, in der der Pianist am Klavier Platz nimmt: Von Beginn seiner Karriere an saß er nie anders denn auf einem von seinem Vater eigens präparierten Klappstuhl, der den Pianisten auf eine niedrigere Sitzhöhe bringt, als sie gewöhnliche Klavierhocker einzunehmen gestatten. Abgesehen von dem kuriosen Gegenstand selbst, dessen Wert für die Imagepflege als erstes Zeichen von Schrulligkeit sicher früh erkannt worden ist - eine Reproduktion ist neuerdings unter www.glenngould-chair.com zu erwerben -, ist die so zustande kommende Sitzhaltung äußerst ungewöhnlich, und kein Klavierlehrbuch würde sie empfehlen: Die Unterarme bilden keine Waagerechte, sondern sind vom Handgelenk zu den Ellbogen hin abwärts geneigt, was für Kraftübertragung und Kontrolle der Finger eigentlich ungünstig sein müsste; die leicht angehobenen Schultern verstärken den Eindruck einer recht unbequemen Position, ganz so, als müsste ein kleines Kind von einem Erwachsenenstuhl aus auf dem Esstisch hantieren.

5 Alle Abbildungen zu Glenn Gould sind Standbilder aus Bruno Monsaingeons Film Glenn Gould. The Alchimist, der 1974 entstanden und 2002 bei EMI Classics als DVD erschienen ist. 


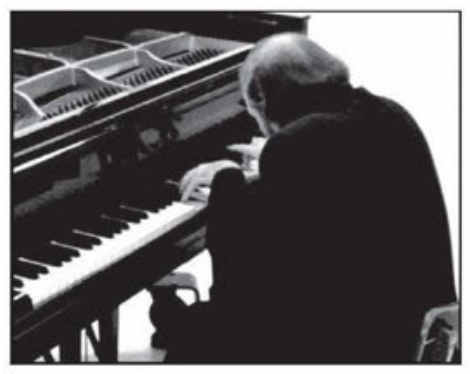

Abb. 4: Glenn Goulds Haltung

Selten wird man erleben, dass ein virtuoser Pianist Gelegenheit findet, eine Hand für überschüssige Nebenbewegungen zu verwenden; Gould aber tut dies. $\mathrm{Zu}$ Beginn eines Contrapunctus aus Bachs KUNST DER FugE etwa vollführt seine Linke, während seine Rechte den einsamen ersten Themeneinsatz spielt, beschwörend anmutende Bewegungen über der Klaviatur, als dirigiere die eine Hand die andere. Sobald der Comes einsetzt, läge es nahe, die hinzugekommene zweite Stimme mit der anderen Hand zu spielen - Gould aber nimmt die Schwierigkeit in Kauf, allein mit der Rechten beide Stimmen auszuführen, um der Linken noch länger zu gestatten, im musikalischen Gewebe unsichtbare Netze zu spinnen.

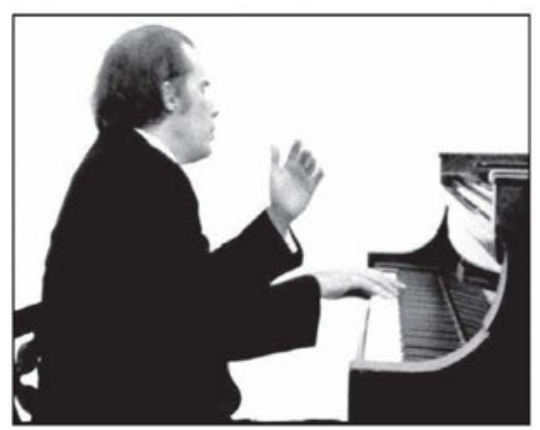

Abb. 5: Glenn Gould »dirigiert»

Auch nachdem der dritte Stimmeneinsatz die Linke doch zurück auf die Tasten geholt hat, bleibt Goulds Kopf weiter in Aktion. Dicht über die Tasten gebeugt, verfolgt er mit größter Aufmerksamkeit sein eigenes Spiel; häufig formen die Lippen rhythmische Plosivlaute, mitunter entsteht sogar hörbarer Gesang, teils in langgezogenen Kantilenen, teils 
wiederum in Staccato-Rhythmen, begleitet von erregtem Kopfschütteln. $\mathrm{Zu}$ sagen, Gould sänge mit, träfe nicht ganz $\mathrm{zu}$, denn vielfach ist sein $\mathrm{Ge}$ sang nicht als Teil des Klavierparts identifizierbar. Es scheint eine Art Meta-Musik zu sein, die aus ihm hervorbricht.

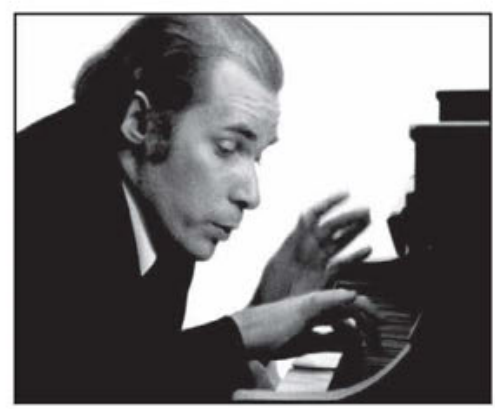

Abb. 6: Glenn Gould »singt«

So lassen sich Goulds Bewegungen keineswegs als unmittelbarer Reflex auf die erklingende Musik beschreiben. Sicher vollzieht er sichtbar die Tempi mit, reagiert auf Themeneinsätze und Modulationen, doch er breitet kein Gefühlsgemälde vor uns aus. Seine emotionale Gestimmtheit bleibt über die Dauer des Spiels weitgehend konstant, wir sehen ihn nicht jubelnd emporgehoben oder in Schmerz niedergedrückt. Keine seiner Bewegungen lässt sich angemessen als Ausdruck eines in der gespielten Musik codierten Gefühls beschreiben, wie es der empfindsamen Programmatik entspräche. ${ }^{6}$ Ebensowenig aber lassen die Aufnahmen die Deutung zu, Gould verwirkliche in seinem Bewegungsverhalten die romantische Idee der Selbstaussprache des Individuums. Was nämlich sagt der Bewegungshabitus, den wir beim klavierspielenden Gould beobachten können, über Gould als Persönlichkeit? Seine Bewegungen beim

6 Gould selber widerspricht der empfindsamen Vorstellung von der Musik als einer Gefühlssprache, wenn er den Pianisten mit einem »Pawlowschen Hund« vergleicht: »Vielleicht, wie Pawlowschen Hunden, schaudert es uns, wenn wir eine vorgehaltene Terzdezime erkennen, und es wird uns warm bei dem sich auflösenden Dominantsextakkord, eben weil wir wissen, daß es das ist, was von uns erwartet wird, eben weil wir zu diesen Reaktionen erzogen worden sind. Vielleicht deshalb, weil wir uns haben beeindrucken lassen von unserer eigenen Fähigkeit zu reagieren. Vielleicht steckt nicht mehr dahinter, als daß wir Gefallen gefunden haben an uns selbst - daß die ganze Wirkung von Musik die Vorführung einer Reflexwirkung ist.« (Gould 1987: 21) 
Spiel würden, erlebte man sie an einem Menschen, der gerade nicht an einem Klavier sitzt, sicher als starke Verhaltensauffälligkeit eingestuft. Sie ähneln am ehesten manischen Tics, die an Menschen zu beobachten sind, die in eine Welt von Wichtigkeiten versunken sind, die für andere unverständlich bleiben müssen. Wenn sie denn eine Sprache darstellten, so wäre dies eine »Privatsprache« und als solche nach Wittgenstein unmöglich, da im wörtlichen Sinne »nicht zu gebrauchen«. Unsere Zweifel an der romantischen Ideologie der »Rest-Bewegungen « als Selbstausdruck werden noch verstärkt, wenn wir im selben Film in einer Interview-Sequenz einen entspannt parlierenden Gould sehen, der sich in Haltung und Gebaren von dem klavierspielenden Gould erheblich unterscheidet.

Vor der Folie des Gouldschen Sich-Bewegens, das sich der Deutung als »Ausdruck von etwas « - seien es musikalisch codierte Leidenschaften, sei es die "Seele« des Spielenden - radikal verweigert, offenbart sich das Ideologische der empfindsamen wie auch der romantischen Vorstellung des Instrumentalmusikers: Für die »Restbewegungen« des Musikers Gould scheint es keine Übersetzung in eine andere Sprache zu geben. Sie unterscheiden sich in ihrer Gestalt, nicht aber in ihrer Bedeutung - denn offenbar haben sie keine. Wir nehmen sie wahr als Zeichen ohne Inhalt, Ausdruck ohne Gehalt. Wollten wir sie Gesten nennen, so wären diese nicht-intentional, denn sie haben ganz offensichtlich weder den Zweck, musikalische Gehalte zu verdeutlichen, noch den, seelische Befindlichkeiten mitzuteilen.

Gould, der im etablierten Musikbetrieb bewusst als Ketzer auftritt und durch seine Verweigerungshaltung die etablierten Rituale der »klassischen Musik « um so sichtbarerer macht, zwingt uns wie kein anderer, das mediale Apriori unserer Wahrnehmungen zu reflektieren. Wir sehen ihn auf nüchternem Bildschirm, aller ästhetisch-ideologischen Vorgaben bereinigt - so scheint es uns. Doch der durch die DVD ermöglichte Blick auf Goulds Spiel ist keineswegs objektiver als die medialen Konstellationen vergangener Jahrhunderte. Wir spulen den Film vor und zurück, betrachten Goulds Hände in Großaufnahme und sezieren so sein Bewegungsverhalten mit kühlem Blick. In unseren geschlossenen Wohnzimmern sitzend, schauen wir in Goulds geschlossenes Tonstudio - von Monade zu Monade. Unter diesen medialen Bedingungen zeugen die »Restbewegungen«, die in der Vergangenheit mit so viel Gehalt beladen worden waren, nur mehr von Fremdheit, Leere und Privatisierung. Hörten wir Gould jedoch im Hintergrund einer Tafelrunde der Frühaufklärung musizieren, würden uns seine Bewegungen gar nicht zu Bewusstsein kommen, vermutlich wüssten wir nicht einmal seinen Namen. Spielte er um 1750 im Kreis unserer Freunde, erkennten wir in ihm eine empfind- 
same Seele und in seinen Bewegungen den Ausdruck davon. Sähen wir ihn als Tastengenie auf einem Podium, schlüge er uns mit seinem Charisma in seinen Bann und wir sähen in seinem Gebaren die Äußerungen eines Subjekts hors du commun.

Der vermeintliche »Gehalt« der »Restbewegungen« erweist sich damit als Effekt der jeweils herrschenden ästhetischen Ideologie und ihrer medialen Produktionsbedingungen. Mit diesen aber ist sie so untrennbar verknüpft, dass der archimedische Punkt jenseits aller kontingenten Bedingungen unerreichbar bleibt. In das, was zu verschiedenen Zeiten ein Musikerlebnis ausmacht, ist die jeweils zulässige »Restbewegung« solcherart integriert, dass sie eben letztlich keinen Rest darstellt. Wenn nämlich die Maßgaben einer rein technisch verstandenen Spielökonomie allein die Dimensionen eines Konzertereignisses nicht hinreichend beschreiben können, dann rückt das, was peripher erschien, zuletzt in dessen Zentrum: Das Sich-Bewegen des Musikers steht im Schnittpunkt der Koordinaten, die das Konzert als Ereignis ausmachen.

Seit Carl Philip Emmanuel Bachs Zugeständnissen an die »anständigen« Gebärden hat keine herrschende Musizierästhetik die »Restbewegungen« gänzlich für unzulässig erklären wollen; im Gegenteil: Die Grenzen der Zulässigkeit wurden beständig erweitert. Diese Karriere verlief im Gleichschritt zu der Entwicklung, mit der sich die bürgerliche Musikkultur zunehmend als »klassische« verstand und ihren zentralen Gegenstand, die Musik, in der Sphäre der Transzendenz verortete. Je kühner nun die Transzendenzbehauptung wurde, die man der nunmehr »klassischen Musik« zugestand, desto mehr Duldung, ja Bedürfnis entstand für den kontrollierten Kontrollverlust am Instrument. Mit jeder die Spielökonomie verlassenden Bewegung aber bringt sich der sterbliche Körper des Musikers in Erinnerung: als ein beständiges Dementi der Geistigkeit klassischer Musik.

Mit den Brechungen der Moderne ist die Transzendenzbehauptung der klassischen Musik unhaltbar geworden, so dass das heutige Fortleben der Kulthandlungen des klassischen Konzertbetriebs zuweilen seltsam anachronistisch anmutet. Glenn Gould zog daraus klug die Konsequenz und markierte für sich allein das Ende einer Tradition. Doch stellen wir uns zum Schluss die Alternative zu dieser radikalen Absage vor: eine fortgesetzte Überbietung der »Restbewegungen « als immer deutlicher werdendes Dementi der Transzendenzbehauptung. Denkbar wäre dieses Dementi in letzter Konsequenz wohl nur so, wie Thomas Mann den letzten Konzertversuch schildert, den Adrian Leverkühn unternimmt:

»Wir sahen Tränen seine Wangen hinunterrinnen und auf die Tasten fallen, die er, naß wie sie waren, in stark dissonantem Akkorde anschlug. Dabei öffnete er den Mund, wie um zu singen, aber nur ein Klagelaut, der mir für immer im Oh- 
re hängengeblieben ist, brach zwischen seinen Lippen hervor; er breitete, über das Instrument gebeugt, die Arme aus, als wollte er sie umfangen, und fiel plötzlich, wie gestoßen, seitlich vom Sessel hinab zu Boden.« (Mann 1990: 663)

Hier vollzieht sich die »Kadenz des ganzen Körpers« im wörtlichen Sinne: der Tod des Musikers am Instrument. Auf diese letzte Konsequenz aber verweist schon die kleinste Schweißperle des Geigers beim MozartKonzert. Die gemeinsame Karriere von Transzendenzbehauptung und »Restbewegung« zeigt, dass diese ein fürs Ganze unverzichtbares Skandalon darstellt: das Et in Arcadia ego der klassischen Musik.

\section{Literatur}

Bach, Carl Philipp Emanuel [1753/1762] (1994): Versuch über die wahre Art das Clavier zu spielen, Hg. von Wolfgang Horn, Kassel: Bärenreiter.

Brandstetter, Gabriele (2002): Die Szene des Virtuosen. Zu einem Topos von Theatralität. In: Hofmannsthal-Jahrbuch 10, S. 213-243.

Couperin, François [1717] (1961): L'art de toucher le clavecin. Die Kunst das Clavecin zu spielen. The Art of playing the Harpsichord, übers. v. Anna Linde/Mevanwy Roberts, Wiesbaden: Breitkopf \& Härtel

Dömling, Wolfgang (1985): Franz Liszt und seine Zeit, Laaber: Laaber.

Gould, Glenn (1987): Rat an eine Abschlußklasse. In: Von Bach bis Boulez. Schriften zur Musik I, Hg. von Tim Page, München, Zürich: Piper, S. 19-25.

Heine, Heinrich (1994): Lutezia. Erster Teil, XXXIII (20. April 1841). In: Werke in vier Bänden, Bd. 3: Schriften über Frankreich, Hg. von Eberhard Galley, Frankfurt am Main, Leipzig: Insel, S. 304- 601.

Hildebrandt, Dieter (2002): Pianoforte. Der Roman des Klaviers im 19. Jahrhundert, München, Wien: DTV.

Kaden, Christian (1993): »Aufbruch in die Illusion«. Kommunikationsstrukturen in der Musik des späteren 18. Jahrhunderts. In: Des Lebens wilder Kreis. Musik im Zivilisationsprozeß, Kassel: Bärenreiter, S. $140-156$.

Kapp, Julius (1969): Niccolo Paganini, Tutzing: Schneider.

Linde, Anna [1717] (1961): Vorwort. In: (Hg.), François Couperin: L'art de toucher le clavecin. Die Kunst das Clavecin zu spielen. The Art of playing the Harpsichord, Wiesbaden: Breitkopf \& Härtel, S. 7 
Mann, Thomas [1947] (1990): Doktor Faustus. Das Leben des deutschen Tonsetzers Adrian Leverkühn, erzählt von einem Freunde, Frankfurt am Main: Fischer.

Mauss, Marcel [1934] (1989): Die Techniken des Körpers. In: Soziologie und Anthropologie 2: Gabentausch, Soziologie und Psychologie, Todesvorstellungen, Körpertechniken, Begriff der Person, Frankfurt am Main: Fischer, S. 199-220.

Mozart, Leopold [1756] (1995): Versuch einer gründlichen Violinschule, Hg. von Greta Moens-Haenen, Kassel u.a.: Bärenreiter.

Schottky, Julius Max [1830] (1909): Paganini's Leben und Treiben als Künstler und Mensch mit unpartheiischer Berücksichtigung der Meinungen seiner Anhänger und Gegner, Prag: Walluf.

Schumann, Robert [1854] (1914) Gesammelte Schriften über Musik und Musiker. Bd. 1-2. Hg. von Martin Kreisig. Leipzig: Breitkopf \& Härtel.

\section{Abbildungsverzeichnis}

Abb. 1: Fuld, Werner (2003): Paganinis Fluch. Die Geschichte einer Legende, Reinbek: Rowohlt, S. 211.

Abb. 2: Karikatur eines Liszt-Auftrittes (Berlin 1842): http://www. onlinekunst.de/oktoberzwei/Franz_von_Liszt_2.htm; Stand 25.12. 2007.

Abb. 3: Fotografie des Pianisten Lang Lang: www.langlang.com; Stand 25.12.2007.

Abb. 4, 5, 6: Filmstills aus: Monsaingeon, Bruno [1974] (2002): Glenn Gould. The Alchimist: EMI (DVD). 